\title{
Strong light-matter coupling in bulk GaN-microcavities with double dielectric mirrors fabricated by two different methods
}

\author{
F. Réveret, ${ }_{1,2, a)}^{1,}$ K. Bejtka, ${ }^{3,4}$ P. R. Edwards, ${ }^{4}$ S. Chenot, ${ }^{3}$ I. R. Sellers, ${ }^{3}$ P. Disseix, ${ }^{1,2}$ \\ A. Vasson, ${ }^{1,2}$ J. Leymarie, ${ }^{1,2}$ J. Y. Duboz, ${ }^{3}$ M. Leroux, ${ }^{3}$ F. Semond, ${ }^{3}$ and R. W. Martin ${ }^{4}$ \\ ${ }^{1}$ Clermont Université, Université Blaise Pascal, LASMEA, BP 10448, F-63000 Clermont-Ferrand, \\ France \\ ${ }^{2}$ CNRS, UMR 6602, LASMEA, F-63177 Aubière, France \\ ${ }^{3}$ CRHEA-CNRS, Rue Bernard Gregory, Parc Sophia Antipolis, 06560 Valbonne, France \\ ${ }^{4}$ Department of Physics, SUPA, University of Strathclyde, Glasgow G4 ONG, United Kingdom
}

(Received 27 May 2010; accepted 15 July 2010; published online 26 August 2010)

\begin{abstract}
Two routes for the fabrication of bulk GaN microcavities embedded between two dielectric mirrors are described, and the optical properties of the microcavities thus obtained are compared. In both cases, the $\mathrm{GaN}$ active layer is grown by molecular beam epitaxy on (111) $\mathrm{Si}$, allowing use of selective etching to remove the substrate. In the first case, a three period $\mathrm{Al}_{0.2} \mathrm{Ga}_{0.8} \mathrm{~N} / \mathrm{AlN}$ Bragg mirror followed by a $\lambda / 2 \mathrm{GaN}$ cavity are grown directly on the Si. In the second case, a crack-free $2 \mu \mathrm{m}$ thick GaN layer is grown, and progressively thinned to a final thickness of $\lambda$. Both devices work in the strong coupling regime at low temperature, as evidenced by angle-dependent reflectivity or transmission experiments. However, strong light-matter coupling in emission at room temperature is observed only for the second one. This is related to the poor optoelectronic quality of the active layer of the first device, due to its growth only $250 \mathrm{~nm}$ above the Si substrate and its related high defect density. The reflectivity spectra of the microcavities are well accounted for by using transfer matrix calculations. (C) 2010 American Institute of Physics. [doi:10.1063/1.3477450]
\end{abstract}

\section{INTRODUCTION}

The large values for exciton binding energies and oscillator strengths found in III-nitride materials offer significant potential for microcavity devices operating at elevated temperature. Recently there have been a number of demonstrations of strong light-matter coupling in III-nitride semiconductor microcavities (MCs) and of important applications of the resulting structures. Strong-coupling in III-nitrides was first reported in MCs fabricated using lower epitaxial $(\mathrm{Al}, \mathrm{Ga}) \mathrm{N}$ layers, in combination with an upper dielectric distributed Bragg reflector (DBR) initially at cryogenic temperature ${ }^{1}$ and then at room temperature using an aluminum top mirror ${ }^{2,3}$ or with a dielectric top DBR. ${ }^{4,5} \mathrm{GaN}$ microcavities with high quality factors $(\mathrm{Q})$ were achieved using near lattice-matched $(\mathrm{Al}, \mathrm{In}) \mathrm{N} /(\mathrm{Al}, \mathrm{Ga}) \mathrm{N}$ mirrors ${ }^{6}$ leading to demonstrations of room temperature strong-coupling with both bulk and quantum-well containing MCs, ${ }^{7,8}$ polariton lasing, ${ }^{9}$ and surface-emitting lasers. ${ }^{10}$

The use of dielectric DBRs, such as silica/zironia $\left(\mathrm{SiO}_{2} / \mathrm{ZrO}_{2}\right)$ or silica/silicon nitride $\left(\mathrm{SiO}_{2} / \mathrm{Si}_{\mathrm{x}} \mathrm{N}_{\mathrm{y}}\right)$ for both upper and lower mirrors of the MC offer a number of advantages over epitaxial DBRs. Limited refractive index contrast in the latter results in relatively narrow high-reflectivity stop bands and there are also limitations on the effective cavity length due to extension of the cavity modes into the DBR. Absorption in the epitaxial mirrors can be problematical in some cases. Furthermore, in the case of $(\mathrm{Al}, \mathrm{Ga}) \mathrm{N}$-based mirrors, cracking is a problem when growing a large number of periods. Alternatively, III-nitride MCs with two dielectric

\footnotetext{
${ }^{a)}$ Electronic mail: francois.reveret@lasmea.univ-bpclermont.fr.
}

DBR mirrors offer the potential for broad stop-bands, higher finesse MCs, reduced cavity length and larger exciton-photon coupling. ${ }^{11}$

There are a number of significant difficulties to overcome, however, in fabricating a high-quality double dielectric DBR III-nitride MC. The inclusion of the second, lower dielectric DBR requires removal of the substrate in order to access the underside of the active region ${ }^{12-18}$ [apart from one demonstration using lateral overgrowth above a stripe patterned dielectric DBR (Ref. 19)]. This can be performed by laser lift-off from a sapphire substrate, ${ }^{13,14}$ etching of $\mathrm{SiC}$ (Ref. 15) or silicon substrates, ${ }^{16,18}$ or combination of grinding, polishing, and etching of a GaN substrate. ${ }^{17}$ Strong light-matter coupling was not demonstrated in any of the above double dielectric MCs but we have recently reported a "double dielectric plus epitaxial" mirror MC fabricated using silicon substrate removal in which the strong coupling regime was verified at $5 \mathrm{~K}$ through angle-dependent reflectivity and transmission measurements. ${ }^{11}$ In the present work, we describe another route for the fabrication of such MCs and compare the optical properties of the cavity thus obtained with those of the cavity reported in Ref. 11.

\section{SAMPLES GROWTH AND CAVITY PROCESSING}

The III-nitride layers were grown by molecular beam epitaxy (MBE) on (111) Si substrates using ammonia as the nitrogen precursor. The fabrication of the first microcavity (sample A) has already been described ${ }^{11}$ but we shall briefly recall it for the sake of completeness. First, a three period $\mathrm{Al}_{0.2} \mathrm{Ga}_{0.8} \mathrm{~N} / \mathrm{AlN}$ Bragg mirror followed by a $\lambda / 2 \mathrm{GaN}$ cavity was directly grown by MBE on a (111) Si substrate. ${ }^{20}$ 

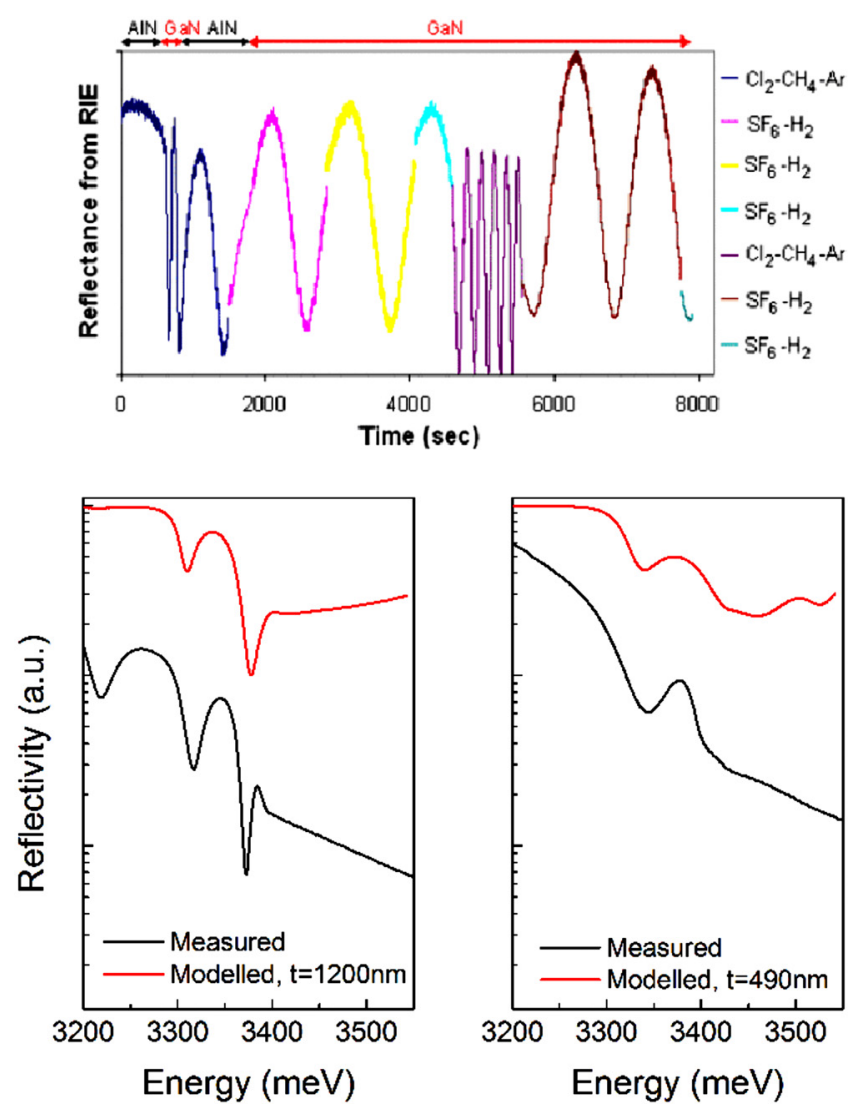

FIG. 1. (Color online) (a) In situ recording of the reflectivity at $633 \mathrm{~nm}$ during the reactive ion thinning of sample B. (b) Ex situ reflectivity spectra and their modeling, measured during the thinning of sample B.

This small number of periods ensures a reasonable material crystalline quality without cracking of the layer. A 7 pair $\mathrm{SiO}_{2} / \mathrm{ZrO}_{2}$ Bragg stack, which will serve as the bottom mirror, was deposited on the top surface and the structure then bonded upside down on a sapphire host substrate. The $\mathrm{Si}$ substrate was removed by mechanical polishing and electron cyclotron etching, with the III-nitride epitaxial stack providing an etch stop. Then the upper mirror was completed by deposition of a three period $\mathrm{SiO}_{2} / \mathrm{ZrO}_{2}$ Bragg stack. ${ }^{11}$

The second fabrication process (sample B) is based on state-of-the-art $\mathrm{GaN}$ on silicon; a $1.5 \mu \mathrm{m}$ thick $\mathrm{GaN}$ layer is grown by $\mathrm{MBE}$ on a $\operatorname{AlN}(40 \mathrm{~nm}) / \mathrm{GaN}(250 \mathrm{~nm}) / \mathrm{AlN}(250$ $\mathrm{nm}$ ) buffer stack deposited on a (111) Si substrate. ${ }^{20}$ An eight pair $\mathrm{SiO}_{2} / \mathrm{Si}_{3} \mathrm{~N}_{4}$ DBR stack, centered at a wavelength of 359 $\mathrm{nm}$, is deposited on top and the structure then cemented upside down on a sapphire substrate. The silicon substrate is then etched away with a $3 \mathrm{HF} / 2 \mathrm{HNO}_{3} / \mathrm{CH}_{3} \mathrm{COOH}$ solution, revealing the $\mathrm{N}$-face of the nitride stack. Then the nitrides were thinned using reactive ion etching by alternating $\mathrm{SF}_{6} / \mathrm{H}_{2}$ and $\mathrm{Cl}_{2} / \mathrm{CH}_{4} /$ Ar-based plasmas. The former is slow and leads to smooth GaN surfaces, while the later is rapid but gives rough surfaces. Indeed, $\mathrm{GaN}$ etch rates were found to be $\approx 8 \mathrm{~nm} / \mathrm{min}$ for $\mathrm{SF}_{6}$ etching and $\approx 60 \mathrm{~nm} / \mathrm{min}$ for $\mathrm{Cl}_{2}$ etching. For AlN, the etch rates are $6 \mathrm{~nm} / \mathrm{min}$ in both cases. Figure 1(a) shows an in situ reflectivity trace at $\lambda=633 \mathrm{~nm}$ of the sample during such thinning, demonstrating the etch rate differences. Knowledge of the etch rates allows the thicknesses to be deduced, and these were also checked later

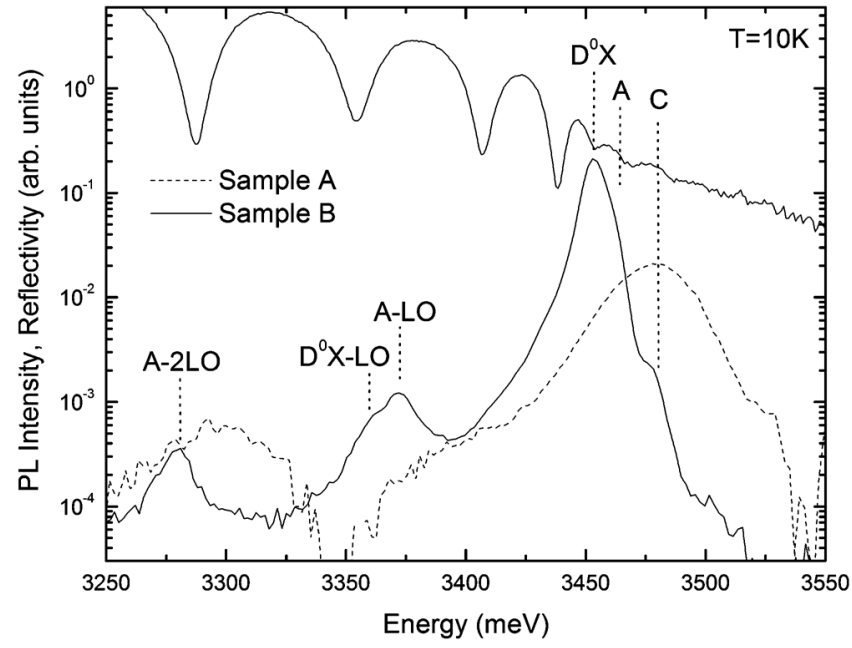

FIG. 2. Reflectivity and PL spectra of the as-grown structure at $10 \mathrm{~K}$ for sample B (solid line) and sample A (dashed line). The intensity scale is not common for the two PL spectra.

using simulations of ex situ experimental reflectivity spectra [Fig. 1(b)]. There is good agreement between both estimations of the GaN thickness. Once the thickness of $140 \mathrm{~nm}$ corresponding to a $\lambda$ cavity with a vacuum wavelength $\left(\lambda_{0}\right)$ of $359 \mathrm{~nm}$ is reached, the microcavity is completed by a three pair $\mathrm{SiO}_{2} / \mathrm{ZrO}_{2}$ upper Bragg mirror.

In the case of the first process, i.e., sample A, the cavity length is defined solely by the epitaxy growth time but this advantage comes with the difficulty of having to use GaN grown very close to the silicon, which is not of the highest quality, because the threading dislocation density reaches $6-8 \times 10^{10} \mathrm{~cm}^{-2}$. In the second process, the active GaN layer is composed of much less defective $\mathrm{GaN}$ (5 $\times 10^{9} \mathrm{~cm}^{-2}$ dislocations) but the control of the etching depth and interface quality is complex and requires the use of in situ reflectometry and an alternation of etch recipes. These differences in the optoelectronic quality of the $\mathrm{GaN}$ can be assessed prior to further processing. Indeed, Fig. 2 shows the $10 \mathrm{~K}$ photoluminescence (PL) spectra of both samples after epitaxy, i.e., still on their silicon substrates. The spectrum of the $1.5 \mu \mathrm{m}$ thick sample B is typical of state-of-the-art GaN on silicon. ${ }^{20}$ It is dominated by neutral donor bound exciton $\left(\mathrm{D}^{0} \mathrm{X}\right.$ at $\left.3453 \mathrm{meV}\right)$ and free $\mathrm{A}$ exciton recombinations, along with their LO phonon replicas. The reflectivity $(\mathrm{R})$ spectrum, also shown, provides resolution of both the A/B and $\mathrm{C}$ free excitons. The low energy of the unresolved $\mathrm{A}$ and B free exciton $(3464 \mathrm{meV}$ at $10 \mathrm{~K})$ is also typical of $\mathrm{GaN}$ on silicon and reflects the tensile stress due to the lower thermal expansion coefficient of $\mathrm{Si}$ relative to $\mathrm{GaN}$. The full width at half maximum (FWHM) of the $\mathrm{D}^{0} \mathrm{X}+\mathrm{A}+\mathrm{B}$ band is $10 \mathrm{meV}$. On the other hand the spectrum of sample $\mathrm{A}$, which is grown on a three period $\mathrm{Al}_{0.2} \mathrm{Ga}_{0.8} \mathrm{~N} / \mathrm{AlN}$ Bragg stack and thus is only $\approx 250 \mathrm{~nm}$ above the $\mathrm{Si}$ substrate, is very broad $(\mathrm{FWHM}=35 \mathrm{meV})$ due to strain inhomogeneities related to the large defect density and also presumably to unintentional silicon doping. It peaks at $3480 \mathrm{meV}$, with the blue shift relative to sample B resulting from the compressive stress imposed by the AlN/(Al,Ga)N Bragg mirror. 

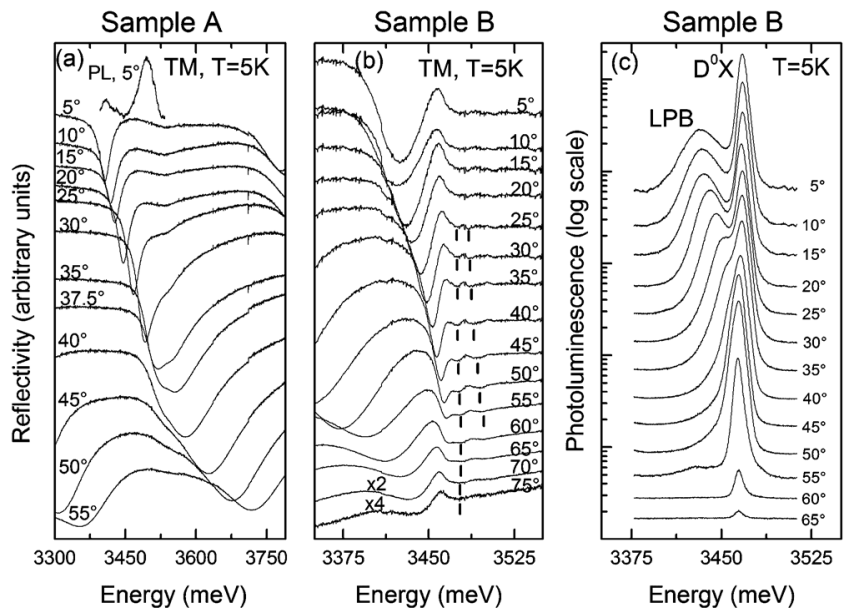

FIG. 3. Angle resolved reflectivity spectra recorded at $5 \mathrm{~K}$ in TM polarization (a) for sample A and (b) sample B and (c) angle resolved PL measured at $5 \mathrm{~K}$ on sample $\mathrm{B}$.

\section{RESULTS FROM THE MICROCAVITIES}

\section{A. Low temperature $(5 \mathrm{~K})$ experiments}

Angle resolved measurements were performed on the fully fabricated microcavities at low $(5 \mathrm{~K})$ and room temperature $(300 \mathrm{~K})$. Some details about the set up are given in Ref. 21. Angle resolved reflectivity spectra recorded at $5 \mathrm{~K}$ for sample A are shown in Fig. 3(a). On the lower angle $\left(5^{\circ}\right)$ spectrum, the optical mode is observed at $3405 \mathrm{meV}$ and a broad excitonic transition is detected at $3530 \pm 10 \mathrm{meV}$. As the incidence angle increases, the anticrossing between photon and exciton is clearly in evidence between $35^{\circ}$ and $37.5^{\circ}$. The Rabi splitting value (labeled $\Omega$ ), which is the minimum difference between lower polariton branch (LPB) and upper polariton branch (UPB), whether or not the middle polariton branch (MPB) is observed, is equal to $43 \pm 3 \mathrm{meV}$. The PL spectrum recorded at $5^{\circ}$ is also shown in this figure. The low energy peak corresponds to the cavity mode in full agreement with the reflectivity measurement and the second peak, centered at $3495 \pm 2 \mathrm{meV}$, is attributed to the neutral bound exciton recombination $\left(\mathrm{D}^{0} \mathrm{X}\right)$. The FWHM of this line, equal to $37 \mathrm{meV}$, is in accordance with the measurement on the as grown structure. PL and R spectra measured from the sample $\mathrm{B}$ at low temperature $(5 \mathrm{~K})$ as a function of angle are also reported in Fig. 3. Figure 3(b) shows the measured reflectivity spectra for various angles of incidence from $5^{\circ}$ to $75^{\circ}$ at $5 \mathrm{~K}$. The photonic mode is only resolved for low angles, with a peak energy of $3422 \pm 2 \mathrm{meV}$ at $5^{\circ}$. For angles greater than $25^{\circ}$ three features can be resolved. The middle and UPBs are visible at $3475 \pm 2$ and $3486 \pm 2 \mathrm{meV}$ for an angle of $30^{\circ}$. The resolution of the $A$ and $B$ free excitons in the reflectivity spectra, with a splitting of around $10 \mathrm{meV}$, indicates a nearly strain-free structure. This is in contrast to the classical situation of micrometer-thick strained $\mathrm{GaN}$ on silicon, where the splitting between $A$ and $B$ excitons is of the order of $1 \mathrm{meV}$ and difficult to observe experimentally. ${ }^{20}$ As the angle of incidence increases beyond $30^{\circ}$, the UPB is pushed to higher energies and can be followed up to an angle of $55^{\circ}$. Absorption within the $\mathrm{GaN}$ coupled with the low finesse of the cavity prevents its observation at higher angles.
An anticrossing is seen for angles near $45^{\circ}$ indicating that the strong-coupling regime (SCR) has been attained. The Rabi splitting value is equal to $34 \pm 3 \mathrm{meV}$. The PL at low temperature and low angle [Fig. 3(c)] shows two features. The peak at $3465 \pm 2 \mathrm{meV}$ corresponds to the bound excitonic PL, narrowed by the effect of the cavity (FWHM reduced to $7.2 \pm 0.5 \mathrm{meV})$. The shift to higher energy with respect to the as-grown structure (Fig. 2) is due to the relief of tensile strain resulting from the removal of the silicon. The energy of the bound exciton indicates a very small strain in the active layer like in free-standing samples. ${ }^{22}$ The lower energy peak is related to the photonic mode and its energy increases with the increase in the incidence angle, merging with the exciton peak near $45^{\circ}$. These emission data show the dispersion of the LPB but no strong coupling due to the dominance of the bound excitons.

The peak energies of the polariton branches at low temperature and their associated FWHM are plotted versus incidence angle in Fig. 4 for both samples. Figure 4(a) compares the reflectivity data of sample A with those from transmission ones, as reported earlier in Ref. 11. The LPB and UPB are clearly identified within both experiments. A higher Rabi splitting is observed in transmission measurements than in reflectivity $(\Omega=56 \mathrm{meV}$ instead of $43 \mathrm{meV})$ as already shown for the case of GaAs microcavities. ${ }^{23}$ The polariton dispersion of sample B is plotted on Fig. 4(b) using the R and PL data. The LPB detected in PL up to $40^{\circ}$ is in good agreement with the reflectivity one. The uncoupled excitonic energies of these samples reveal a high compressive strain of $27 \pm 3 \mathrm{kbar}$ in sample $\mathrm{A}$ and a slight tensile strain of $4 \pm 2$ kbar in sample B.

The line width of the polaritonic modes, extracted from the fitting of the curves (reflectivity dips or PL lines), are plotted in Figs. 4(c) and 4(d) for sample A and B, respectively. The photonic line width $\left(\gamma_{\mathrm{ph}} \sim 32 \mathrm{meV}\right)$ of these cavities corresponds to a quality factor (Q) of about 110 . This value is in rather good agreement with the $Q$ value of 120 obtained from simulations within the transfer matrix formalism (not shown here). At the SCR resonance, both polariton branches have a half-photon half-exciton nature and are expected to have a similar FWHM, ${ }^{24}$ as observed on these figures. In Ref. 25 the polaritonic line width is shown to be $\left(\sigma+\gamma_{\mathrm{ph}}\right) / 2$ for $\Omega \sim \sigma$ and $\left(\gamma+\gamma_{\mathrm{ph}}\right) / 2$ when $\Omega \gg \sigma$; where $\gamma$ and $\sigma$ correspond to the homogeneous and inhomogeneous excitonic broadenings, respectively. Sample A corresponds to the first case, $\Omega \sim \sigma(\sigma=40 \mathrm{meV})$, thus the polaritonic line width should be $\left(\sigma+\gamma_{\mathrm{ph}}\right) / 2=36 \mathrm{meV}$. The second case is observed for sample B $(\sigma=11 \mathrm{meV}$, such that $\Omega \gg \sigma)$ and the predicted line width is $\left(\gamma+\gamma_{\mathrm{ph}}\right) / 2=16 \mathrm{meV}$. The experimental FWHMs at resonance are equal to $45 \pm 10 \mathrm{meV}$ at $37.5^{\circ}$ for sample $\mathrm{A}$ and $11 \pm 5 \mathrm{meV}$ at $45^{\circ}$ for sample B. The high inhomogeneous excitonic broadening of sample A $(\Omega$ $\sim \sigma$ ) limits the observation of the SCR to low temperature for this structure. For sample A [Fig. 4(c)], the UPB line width at lower angles corresponds to the excitonic broadening. At $5 \mathrm{~K}$, due to the large inhomogeneous broadening, only a wide excitonic dip is detected (around $60 \mathrm{meV}$ ) which includes contributions from both $\mathrm{A}$ and $\mathrm{B}$ excitons. At high angles, the increases in the FWHMs are ascribable to the 
Sample A
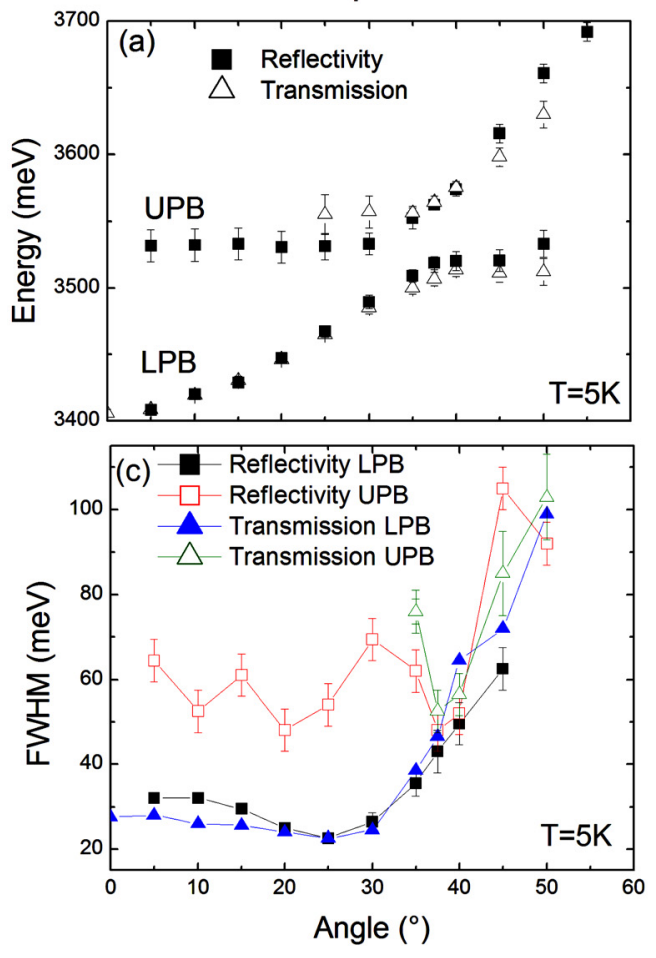

Sample B
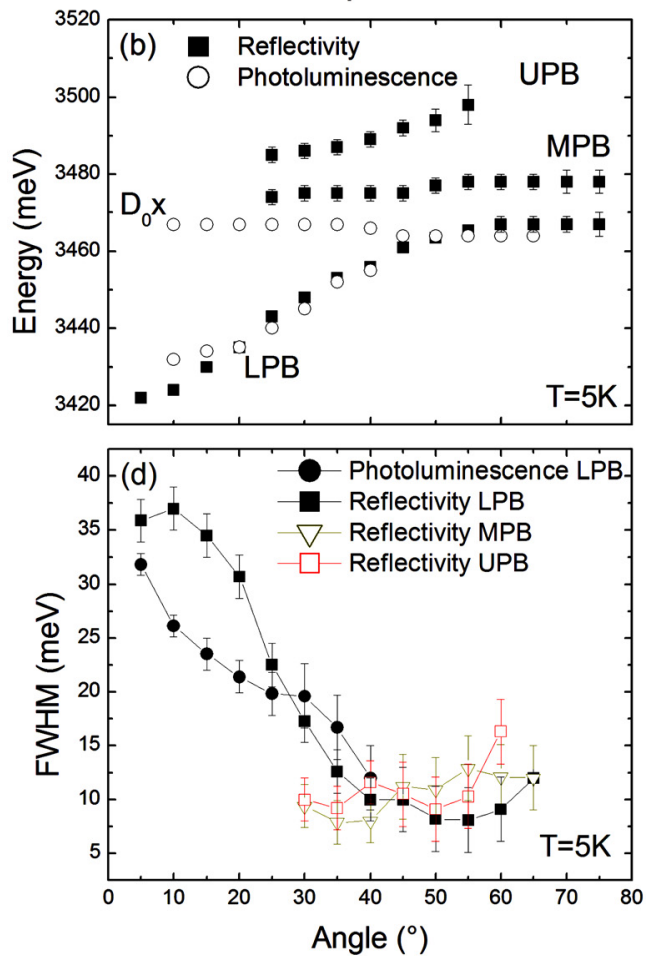

FIG. 4. (Color online) The dispersion of the polaritonic modes and their associated line width at $5 \mathrm{~K}$ for sample $\mathrm{A}[(\mathrm{a})$-(c)] and B [(b)-(d)], deduced using the data in Fig. 3.

large band to band absorption of the active layer. The higher material quality of $\mathrm{GaN}$ allows resolution of both $\mathrm{A}$ and $\mathrm{B}$ excitonic lines in reflectivity, as reported in Fig. 4(b). The measurements show a very low inhomogeneous broadening, equal to $11 \mathrm{meV}$. However, in this cavity, the huge absorption of this thicker GaN active layer prevents the detection of the polaritonic modes beyond $65^{\circ}$.

\section{B. Room temperature $(300 \mathrm{~K})$ experiments}

Angle resolved measurements for both samples at room temperature are reported in Fig. 5. As expected, reflectivity spectra measured from sample A exhibit a weak coupling
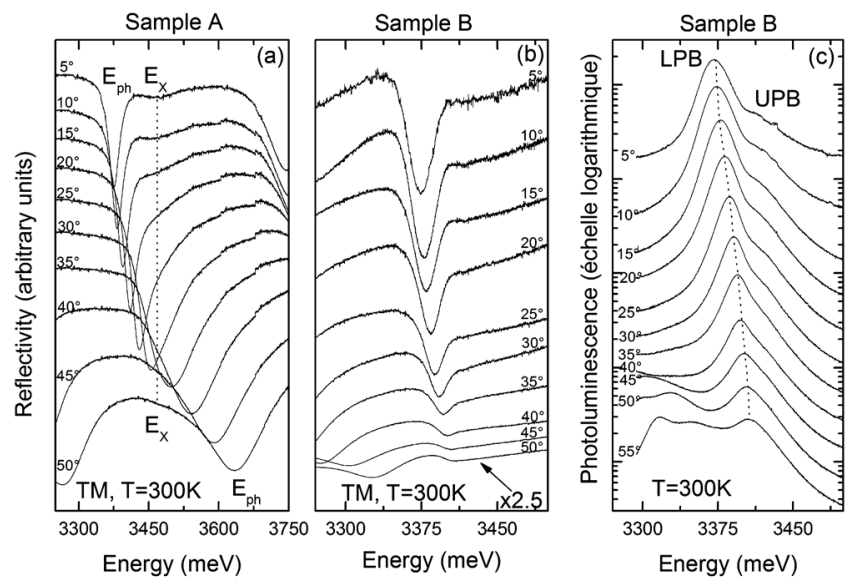

FIG. 5. Angle resolved reflectivity spectra recorded at room temperature in TM polarization (a) for sample A and (b) sample B and (c) angle resolved PL measured at $300 \mathrm{~K}$ on sample B. regime (WCR) as seen in Fig. 5(a). At room temperature, the contribution of the excitonic homogeneous broadening $[\gamma$ $=15 \mathrm{meV}$ at $300 \mathrm{~K}$ (Ref. 22)] leads to a global excitonic line width $(\Gamma)$ around $52 \mathrm{meV}$. This result is in agreement with the experimental study of Christmann et al., ${ }^{8}$ where the transition from SCR to WCR is observed for $\Gamma>\Omega$. For sample B [Fig. 5(b)], the evolution of the LPB through the reflectivity measurements is clearly in agreement with a SCR regime. The SCR at room temperature is also demonstrated by the angle resolved PL spectra measured at $300 \mathrm{~K}$, as shown in Fig. 5(c). At room temperature the PL is dominated by recombination of the $\mathrm{A}$ and $\mathrm{B}$ free excitons, not resolved from each other due to the thermal broadening. At the angle of $5^{\circ}$ the photonic and excitonic modes can be clearly resolved and the evolution of the LPB (UPB) can be followed with increasing angle up to $55^{\circ}\left(40^{\circ}\right)$. As in Fig. 4 , the peak energies and the FWHMs are estimated in a systematic way by fitting the PL emission with two Gaussian functions.

Figure 6(a) shows the peak energies of the photonic and excitonic modes of sample A plotted as a function of angle. With increasing angle, the photonic mode moves to the high energy side and crosses the excitonic energies. The polaritonic dispersion of sample B is reported on Fig. 6(b). An anticrossing is seen for angles near $40^{\circ}$ and a Rabi splitting of $24 \pm 3 \mathrm{meV}$ is estimated from the PL data. The LPB energy measured on the reflectivity spectra is in good agreement with those deduced from PL. The photonic and excitonic line widths deduced from reflectivity spectra of sample A are shown on Fig. 6(c). The FWHM of the excitonic line is about $90 \mathrm{meV}$, corresponding to the unresolved $\mathrm{A}$ and $\mathrm{B}$ excitons and their associated inhomogeneous and homoge- 


\section{Sample A}
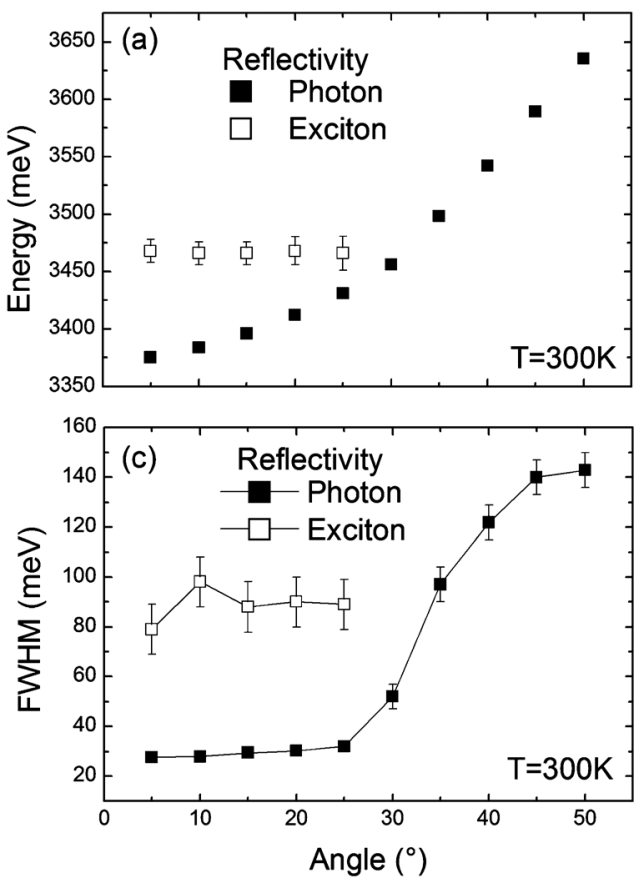

Sample B
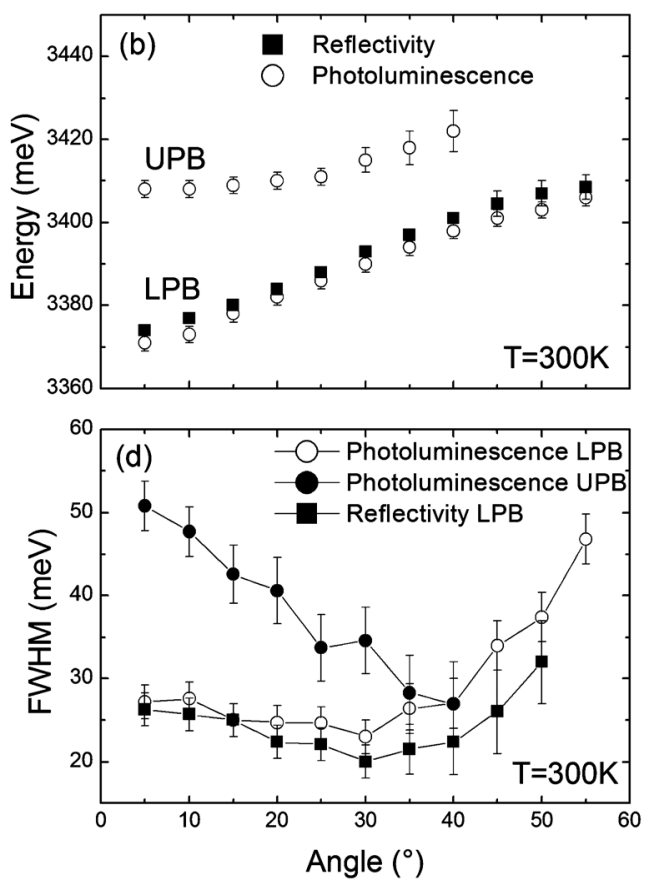

FIG. 6. The evolution of the photonic and excitonic modes (a) for sample A and the polaritonic dispersion for (b) sample B; and the corresponding FWHMs vs incidence angle [(c)-(d)] for both samples, deduced from Fig. 5.

neous broadenings. The photonic line width has the typical evolution observed for the WCR. ${ }^{18}$ It remains constant in the energy range where GaN is optically transparent and increases for the energies corresponding to the excitonic and band to band absorption of the active layer (beyond $25^{\circ}$ ). For sample B, the angle dependence of the FWHMs of both polariton branches are plotted in Fig. 6(d). The LPB FWHM shows a slight decrease for angles up to $30^{\circ}$ followed by a steeper increase, whereas the UPB narrows by a factor of two for increasing angles from $5^{\circ}$ to $40^{\circ}$. As previously observed at low temperature and in the case of a SCR, the FWHMs of the polaritonic modes are identical at the resonance angle. The $300 \mathrm{~K}$ polaritonic line width, equal to $27 \pm 5 \mathrm{meV}$ is in good agreement with the expected value, $\left(\gamma+\gamma_{\mathrm{ph}}\right) / 2=23.5 \mathrm{meV}$.

The reflectivity spectra at the resonance positions for samples A and B for both temperatures are compared with simulations performed using the transfer-matrix formalism, in Fig. 7. Details of the transfer-matrix method are described elsewhere. ${ }^{26}$ From 5 to $300 \mathrm{~K}$ and for each sample, the only parameters changed in the simulations are the excitonic homogeneous broadening (from 0.1 to $15 \mathrm{meV}$ ) and the excitonic energies which vary as a function of temperature. The simulated spectra are in good agreement with the measurements. The strong difference of strain experienced by the active layer of the two samples is clearly in evidence in these spectra. From Fig. 4, the MPB branch energy of sample B is $3475 \mathrm{meV}$ for negative detuning. This shows that the active GaN layer is almost fully relaxed (the A exciton energy of relaxed $\mathrm{GaN}$ is $3477 \mathrm{meV}$ ). On the other hand, the excitonic energy of sample $A$ is $3535 \pm 10 \mathrm{meV}$ for negative detuning. ${ }^{11}$ This strong experimental blue shift of the GaN excitonic energies $(60 \pm 10 \mathrm{meV})$ after $\mathrm{Si}$ removal is due to the compressive stress caused by the three period DBR.

Although the active layer of sample $B$ ( $\lambda$ thickness) is two times thicker than that of sample $A(\lambda / 2$ thickness), the thinnest cavity exhibits the largest Rabi splitting, with values of 43 and $34 \mathrm{meV}$ evaluated for samples A and B, respectively, using the $5 \mathrm{~K}$ reflectivity experiments. The following arguments can qualitatively explain this counter-intuitive result:

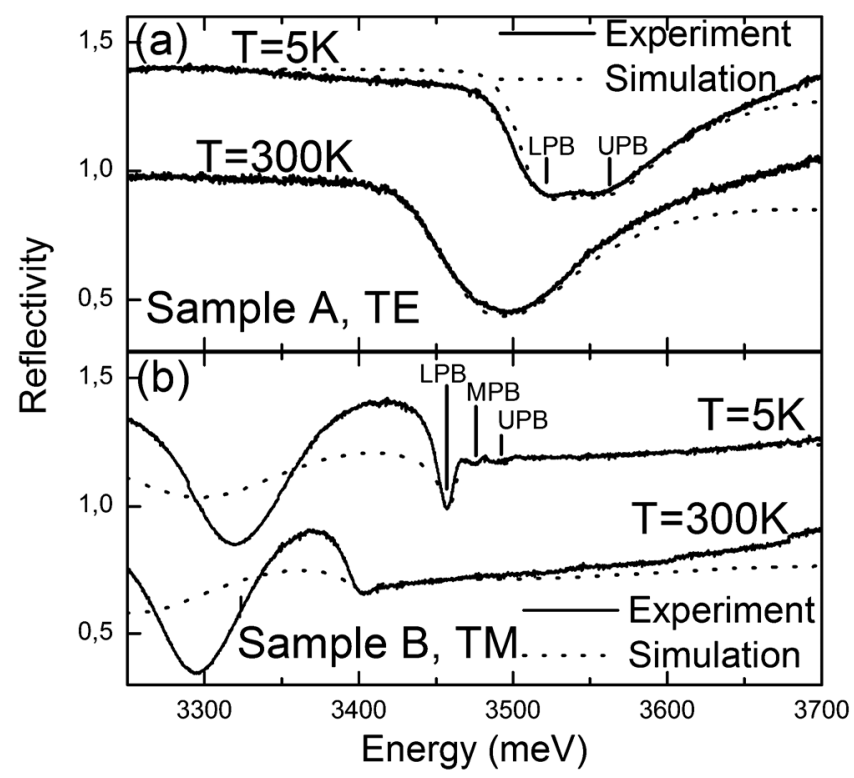

FIG. 7. Experimental reflectivity spectra at resonance angle (solid line) at low and room temperature. The measurements are realized in TE and TM polarization, respectively, for (a) sample A and (b) sample B. The calculated spectra are shown by the dotted lines. 
the large A-B splitting (18 meV instead of $11 \mathrm{meV}$ for sample A), tentatively attributed to the high compressive stress experienced by the $\lambda / 2 \mathrm{GaN}$ active layer in sample $\mathrm{A},{ }^{21}$ artificially increases the energy difference between the LPB and UPB features.

(ii) The oscillator strength values used for the simulations presented in Fig. 7 are $30000(\mathrm{meV})^{2}(\mathrm{~A}$ and B excitons for sample A), $18000(\mathrm{meV})^{2}$ (A exciton, sample B), and $12000(\mathrm{meV})^{2}$ (B exciton, sample $\mathrm{B})$; the accuracy of these values is estimated to be about $10 \%$ and is reduced by the significant inhomogeneous broadening of both samples through the fitting procedure. The fact that the $\mathrm{B}$ exciton oscillator strength is equal to of the A exciton for the highly compressively stressed sample A is in agreement with the theory and with previous measurements on high quality bulk GaN. ${ }^{22}$ However, the values for sample A are larger than those for sample B, which is almost relaxed. This point could be explained by the high compressive stress which modifies the effective mass of the conduction and valence bands and thus induces an increase in the excitonic oscillator strengths. In Ref. 22, a slight increase is also reported but the stress experienced by the samples in that report is less than in sample A.

(iii) As opposed to the situation in sample B, the magnitudes of the photonic and excitonic broadening are similar in sample A $(32 \mathrm{meV}$ and $40 \mathrm{meV}$, respectively), which induces the configuration for an increased Rabi splitting. It has been shown that the latter tends to a maximum value when photonic and excitonic broadenings are the same. ${ }^{26}$

Despite the high optical quality of the GaN active layer of the sample B, the weak photon lifetime $(\mathrm{Q}$ $=110$ ) in the cavity prevents the observation of the polariton lasing. ${ }^{27}$

In summary, strong light-matter coupling has been demonstrated in bulk GaN-microcavities with double dielectric mirrors fabricated by two different methods. The first fabrication approach involves growing the active layer on a three period $\mathrm{Al}(\mathrm{Ga}) \mathrm{N}$ DBR. The SCR is observed at $5 \mathrm{~K}$ using reflectivity and transmission measurements with Rabi splittings, respectively, equal to 43 and $56 \mathrm{meV}$. However, the large excitonic inhomogeneous line width prevents observation of the SCR at room temperature. The second fabrication procedure involves removal of the silicon substrate and controlled thinning of a thick GaN layer, allowed an ultrathin GaN microcavity to be obtained without a buffer layer being embedded between two dielectric DBRs. A very low excitonic inhomogeneous broadening of $11 \mathrm{meV}$ shows the high quality of the active layer. The anticrossing between the A and $\mathrm{B}$ excitons and the photonic mode is observed at $5 \mathrm{~K}$ and polaritonic emission is in evidence at room temperature. This study highlights the importance of the high quality of the active layer to achieve a device working in the SCR at room temperature.
Funding from the EU projects STIMSCAT (STREP Contract No. 517769) and CLERMONT2 (Grant No. MRTN-CT2003-503677) and from the UK EPSRC is acknowledged.

${ }^{1}$ N. Antoine-Vincent, F. Natali, D. Byrne, A. Vasson, P. Disseix, J. Leymarie, M. Leroux, F. Semond, and J. Massies, Phys. Rev. B 68, 153313 (2003).

${ }^{2}$ F. Semond, I. R. Sellers, F. Natali, D. Byrne, M. Leroux, J. Massies, N. Ollier, J. Leymarie, P. Disseix, and A. Vasson, Appl. Phys. Lett. 87, 021102 (2005).

${ }^{3}$ I. R. Sellers, F. Semond, M. Leroux, J. Massies, P. Disseix, A.-L. Henneghien, J. Leymarie, and A. Vasson, Phys. Rev. B 73, 033304 (2006)

${ }^{4}$ I. R. Sellers, F. Semond, M. Leroux, J. Massies, M. Zamfirescu, F. Stokker-Cheregi, M. Gurioli, A. Vinattieri, M. Colocci, A. Tahraoui, and A. A. Khalifa, Phys. Rev. B 74, 193308 (2006).

${ }^{5}$ A. Alyamani, D. Sanvitto, A. A. Khalifa, M. S. Skolnick, T. Wang, F. Ranalli, P. J. Parbrook, A. Tahraoui, and R. Airey, J. Appl. Phys. 101, 093110 (2007).

${ }^{6}$ J.-F. Carlin, J. Dorsaz, E. Feltin, R. Butté, N. Grandjean, M. Ilegems, and M. Laügt, Appl. Phys. Lett. 86, 031107 (2005).

${ }^{7}$ R. Butté, G. Christmann, E. Feltin, J.-F. Carlin, M. Mosca, M. Ilegems, and N. Grandjean, Phys. Rev. B 73, 033315 (2006).

${ }^{8}$ G. Christmann, R. Butté, E. Feltin, J.-F. Carlin, and N. Grandjean, Phys. Rev. B 73, 153305 (2006).

${ }^{9}$ S. Christopoulos, G. Baldassarri Höger von Högersthal, A. Grundy, P. G. Lagoudakis, A. V. Kavokin, J. J. Baumberg, G. Christmann, R. Butté, E. Feltin, J.-F. Carlin, and N. Grandjean, Phys. Rev. Lett. 98, 126405 (2007).

${ }^{10}$ E. Feltin, G. Christmann, J. Dorsaz, A. Castiglia, J.-F. Carlin, R. Butte', N. Grandjean, S. Christopoulos, G. Baldassarri Höger von Högersthal, A. J. D. Grundy, P. G. Lagoudakis, and J. J. Baumberg, Electron. Lett. 43, 924 (2007).

${ }^{11}$ K. Bejtka, F. Réveret, R. W. Martin, P. R. Edwards, A. Vasson, J. Leymarie, I. R. Sellers, J. Y. Duboz, M. Leroux, and F. Semond, Appl. Phys. Lett. 92, 241105 (2008).

${ }^{12}$ H. Benisty, H. De Neve, and C. Weisbuch, IEEE J. Quantum Electron. 34, 1612 (1998).

${ }^{13}$ Y. K. Song, H. Zhou, M. Diagne, A. V. Nurmikko, R. P. Schneider, Jr., C. P. Kuo, M. R. Krames, R. S. Kern, C. Carter-Coman, and F. A. Kish, Appl. Phys. Lett. 76, 1662 (2000)

${ }^{14}$ R. W. Martin, P. R. Edwards, H.-S. Kim, K.-S. Kim, T. Kim, I. M. Watson, M. D. Dawson, Y. Cho, T. Sands, and N. W. Cheung, Appl. Phys. Lett. 79, 3029 (2001).

${ }^{15}$ T. Tawara, H. Gotoh, T. Akasaka, N. Kobayashi, and T. Saitoh, Appl. Phys. Lett. 83, 830 (2003).

${ }^{16}$ T. K. Kim, S. S. Yang, J. K. Hong, and G. M. Yang, Appl. Phys. Lett. 89, 041129 (2006).

${ }^{17}$ F. Rizzi, P. R. Edwards, K. Bejtka, F. Semond, X. N. Kang, G. Y. Zhang, E. Gu, M. D. Dawson, I. M. Watson, and R. W. Martin, Appl. Phys. Lett. 90, 111112 (2007).

${ }^{18}$ K. Bejtka, P. R. Edwards, R. W. Martin, F. Réveret, A. Vasson, J. Leymarie, I. R. Sellers, M. Leroux, and F. Semond, Semicond. Sci. Technol. 23, 045008 (2008)

${ }^{19}$ R. W. Martin, P. R. Edwards, K. P. O'Donnell, M. D. Dawson, C.-W. Jeon, C. Liu, G. R. Rice, and I. M. Watson, Phys. Status Solidi A 201, 665 (2004).

${ }^{20}$ F. Semond, Y. Cordier, N. Grandjean, F. Natali, B. Damilano, S. Vézian, and J. Massies, Phys. Status Solidi A 188, 501 (2001).

${ }^{21}$ F. Réveret, P. Disseix, J. Leymarie, A. Vasson, F. Semond, M. Leroux, and J. Massies, Phys. Rev. B 77, 195303 (2008).

${ }^{22}$ O. Aoudé, P. Disseix, J. Leymarie, A. Vasson, M. Leroux, E. Aujol, B. Beaumont, A. Trassoudaine, and Y. André, Phys. Rev. B 77, 045206 (2008).

${ }^{23}$ R. P. Stanley, R. Houdré, C. Weisbuch, U. Oesterle, and M. Ilegems, Phys. Rev. B 53, 10995 (1996).

${ }^{24}$ C. Ell, J. Prineas, T. R. Nelson, Jr., S. Park, H. M. Gibbs, G. Khitrova, S. W. Koch, and R. Houdré, Phys. Rev. Lett. 80, 4795 (1998).

${ }^{25}$ R. Houdré, R. P. Stanley, and M. Ilegems, Phys. Rev. A 53, 2711 (1996).

${ }^{26}$ F. Réveret, P. Disseix, J. Leymarie, A. Vasson, F. Semond, M. Leroux, and J. Massies, Solid State Commun. 150, 122 (2010).

${ }^{27}$ D. Solnyshkov and G. Malpuech, Superlattices Microstruct. 41, 279 (2007). 